\title{
PAPR Reduction in OFDM using Active and Non-Active Channels
}

\author{
Ketul Patel ${ }^{1}$, Swapnil Bhavsar ${ }^{2}$, Mihir Patel ${ }^{3}$, Vijay Prakash Singh ${ }^{4}$ \\ ${ }^{I}$ (Electronics \& Communication, BHABHA Engg. \& Research Institute,Bhopal,India) \\ ${ }^{2}$ (Electronics \& Communication, BHABHA Engg. \& Research Institute,Bhopal,India) \\ 3 (Electronics \& Communication, RKDF Institute of Science \& Technology, Bhopal, India) \\ ${ }^{4}$ (Electronics \& Communication, BHABHA Engg. \& Research Institute,Bhopal,India)
}

\begin{abstract}
According to the request of advance communication field there should be high data rate in addition to both power efficiency and lower bit error rate. This request of high data rate can be achieved by the multi carrier modulation scheme using the OFDM technique. But the great drawback of the OFDM technique is its high peak to average power ratio (PAPR). High PAPR reduces OFDM signals by driving the analog amplifier to work in the nonlinear region, changing this way the signal and making the amplifier to consume more power. To reduce the PAPR methods exist which adjust or present new signals to battle large signal peaks. The methods which use data carrying channels are called active channel methods and which use redundant channel are called non active channel methods. This work deals with reduction of the PAPR of OFDM signal using both Active and Non Active Channels. Clipping technique has been applied to active channels and Tone reservation has been applied to Non Active Channels. By using both channels we can get considerable reduction in PAPR.
\end{abstract}

Keywords:Orthogonal Frequency Division Multiplexing (OFDM), Peak to Average Power Ratio (PAPR), Power Amplifier (PA).

\section{INTRODUCTION}

The necessity of high data rate services has been increasing very quickly and there is no delay in sight. We know that the data transmission contains both wired and wireless medium. Often, these services need very reliable data transmission over very merciless environment. Most of these transmission systems experience much deficiency such as large attenuation, time variance, multipath, interference, noise, nonlinearities and must meet the finite constraints like power limitation and cost factor. One physical layer technique that has got a lot of reputations due to its roughness in dealing with these impairments is multicarrier modulation technique. In multicarrier modulation, the most commonly used technique is Orthogonal Frequency Division Multiplexing (OFDM); it has in latest times become very popular in wireless communication.

Unfortunately the major drawback of OFDM transmission is its large envelope fluctuation which is measured as Peak to Average Power Ratio (PAPR). Since power amplifier (PA) is used at the transmitter, so as to operate in a perfectly linear region the operating power must lies lower the available power. For reduction of this PAPR lot of techniques have been developed. All of the techniques have some sort of rewards and drawbacks. Clipping and Filtering is one of the basic technique in which some part of transmitted signal suffers into distortion. Also the Coding scheme reduces the data rate which is unwanted. If we consider Tone Reservation (TR) technique it also allows the data rate loss with more probable of increasing power. Again the techniques like Tone Injection (TI) and the Active Constellation Extension (ACE) having an attitude of increasing power will be undesirable in case of power constraint environment. From the Partial Transmit Sequence (PTS) and Selected Mapping (SLM) technique, the PTS technique has more difficulty than that of SLM technique. But most of these methods are unable to achieve a large reduction in PAPR. So PAPR of OFDM signal must be reduced enough to make proper working of the power amplifier.

\section{INTRODUCTION OF OFDM}

Multi-carrier modulation, in particular Orthogonal Frequency Division Multiplexing (OFDM), has been successfully applied to a wide variety of digital communications applications over the past several years. OFDM is frequently referred to as multi-carrier modulation because it transmits signals over multiple subcarriers simultaneously, enabling it to boost WLAN data rates. It is based on the Fast Fourier transform (FFT) concept, which allows the multiple subcarriers to overlap yet maintain their integrity. Because these subcarriers are sent at lower data rates, OFDM systems perform robustly in severe multi-path environments. As a result, this modulation approach is being widely adopted in WLAN implementations.

The use of Frequency Division Multiplexing (FDM) goes back over a century, where more than one low rate signal, such as telegraph, was approved over a comparatively wide bandwidth channel using a split carrier frequency for every signal. To make easy partition of the signals at the receiver, the Carrier frequencies 
were spaced satisfactorily far apart so that the signal spectra did not extend beyond. Empty spectral regions between the signals guaranteed that they could be alienated with readily possible filters. The resulting spectral efficiency was therefore quite small.

Frequency division multiplexing (FDM) is a technology that transmits multiple signals concurrently over a single transmission path, such as a cable or wireless system. Each signal travels within its possess exclusive frequency range (carrier), which is modulated by the data. Accessible bandwidth is divided in multiple subcarriers which are not overlapping. Orthogonal FDM's (OFDM) spread spectrum technique distributes the data over a large number of carriers that are spaced apart at specific frequencies. This spacing provides the "orthogonality" in this technique which prevents the demodulators from seeing frequencies other than their own. The benefits of OFDM are high spectral efficiency, resiliency to RF interfering, and lower multipath distortion.

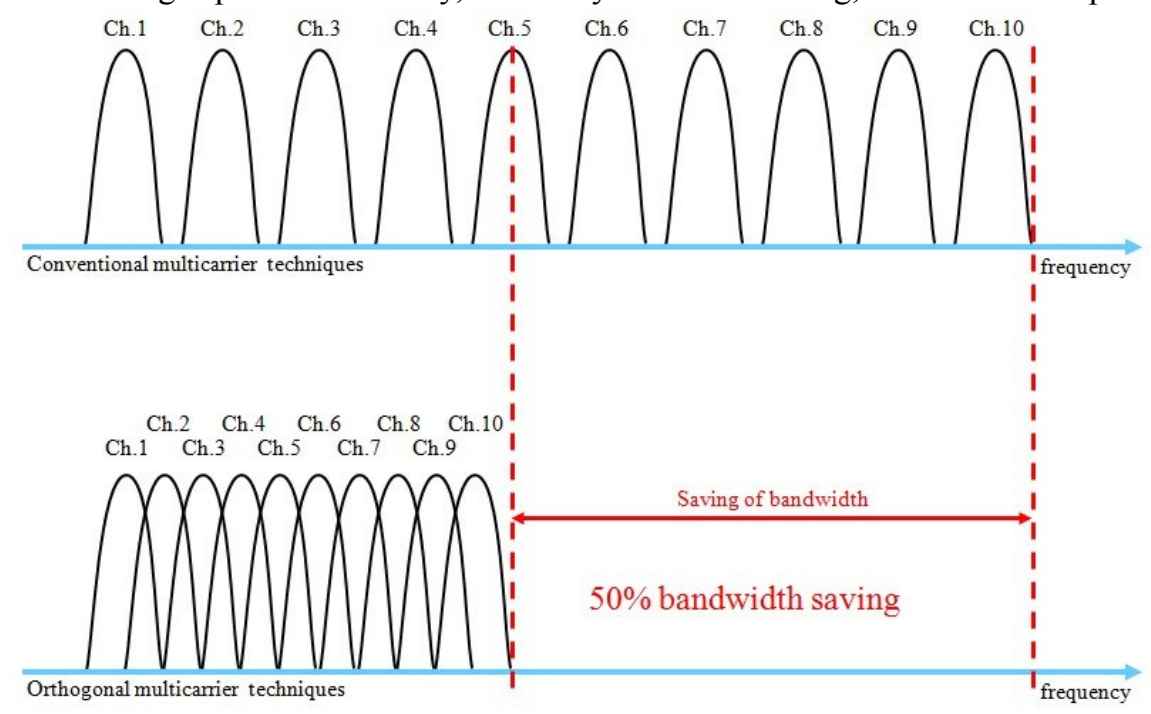

Fig 1: Conventional and Orthogonal multicarrier techniques

To generate the OFDM signal, first the high data rate signal is given to serial to parallel convertor. Each signal is modulated individually by separate subcarrier and then given to IFFT block. The IFFT will combine each separately modulated carrier and clear to serial form. This process will be repeated at the receiver side to get the original signal back. Instead of carrying separate messages, the different frequency carriers can carry different bits of a single higher rate message. The source may be in such a parallel format, or a serial source can be presented to a serial-to-parallel converter whose output is fed to the multiple carriers.

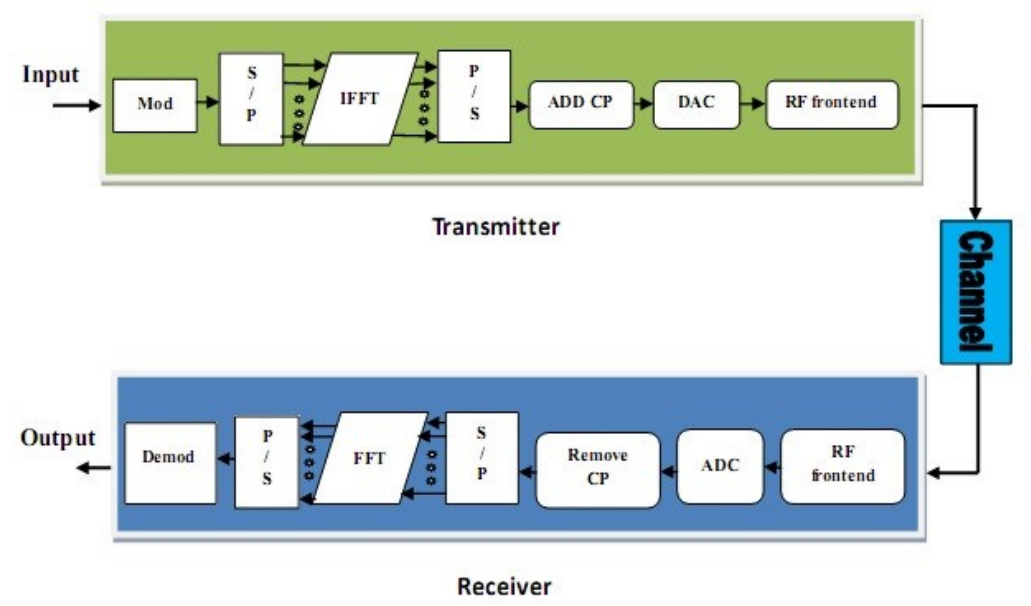

Fig 2: OFDM Block Diagram

An OFDM symbol involves of $\mathrm{N}$ subcarriers by the frequency spacing of $\Delta \mathrm{f}$. Thus, the total bandwidth $\mathrm{B}$ will be alienated into $\mathrm{N}$ equally spaced subcarriers. And all the subcarriers are orthogonal to each other within a time interval of length $\mathrm{T}=\frac{1}{\Delta f}$. Each subcarrier can be modulated separately with the composite modulation symbol $\mathrm{X}_{\mathrm{m}, \mathrm{n}}$, where ' $\mathrm{m}$ ' is a time index and ' $\mathrm{n}$ ' is a subcarrier index. Then within the time interval $\mathrm{T}$ the subsequent signal of the $\mathrm{m}$-th OFDM block period can be described by equation (1) as: 
$\mathrm{X}_{\mathrm{m}}(\mathrm{t})=\frac{1}{\sqrt{N}} \sum_{\mathrm{n}=0}^{N-1} X_{m, n} g_{n}(t-m T)$

Where, $g_{n}(\mathrm{t})$ is distinct through equation (2).

$g_{n}(\mathrm{t})=\left\{\begin{array}{cc}\exp (j 2 \pi n \Delta f t), & 0 \leq t \leq T \\ 0, & \text { else }\end{array}\right.$

Where, $g_{n}(\mathrm{t})$ is a rectangular pulse practical to each subcarrier. The total continuous time signal $\mathrm{X}(\mathrm{t})$ consisting of all the OFDM blocks is given by equation (3).

$\mathrm{X}(\mathrm{t})=\frac{1}{\sqrt{N}} \sum_{m=0}^{\infty} \sum_{n=0}^{N-1} X_{m, n} g_{n}(t-m T)$

Now, consider a single OFDM symbol $(\mathrm{m}=0)$ without loss of simplification. This can be shown because there is no over lie between different OFDM symbols.

Since $m=0, X_{m, n}$ can be replaced by $X_{n}$. Then, the OFDM signal can be described as follows.

$\mathrm{X}(\mathrm{t})=\frac{1}{\sqrt{N}} \sum_{n=0}^{N-1} X_{n} e^{j 2 \pi n \Delta f t}$

If the bandwidth of the OFDM signal is $\mathrm{B}=\mathrm{N} \times \Delta \mathrm{f}$ and the signal $\mathrm{X}(\mathrm{t})$ is sampled by the sampling time of $\Delta \mathrm{t}=$ $\frac{1}{B}=\frac{1}{N \Delta f}$, then the OFDM signal is in discrete time form and can be written as shown in equation (5).

$\mathrm{X}_{\mathrm{k}}=\frac{1}{\sqrt{N}} \sum_{n=0}^{N-1} X_{n} e^{j 2 \pi k n / N}, \mathrm{k}=0,1, \ldots, \mathrm{N}-1$

where, ' $n$ ' denotes the index in frequency domain and $X_{n}$ is the complex symbol in frequency domain. In addition, equation (5) can be expressed using the IFFT.

\section{INTRODUCTION OF PAPR}

When in time domains all the $\mathrm{N}$ subcarriers are added up usefully, they construct a peak power that is $\mathrm{N}$ times greater than the average power of the signal. The PAPR is calculated by the following equation

PAPR $=\frac{\max \left(x^{2}(t)\right)}{\operatorname{mean}\left(x^{2}(t)\right)}$

Where $\mathrm{x}(\mathrm{t})$ is the amplitude of the signal.

The peak power of the OFDM signal, regarding the worst case when all the subcarriers are added-up constructively, is the sum of all the $\mathrm{N}$ subcarriers: $1 \mathrm{~N}=\mathrm{N}$. The mean power of the OFDM signal is the sum of all the values of the signal, which is actually $\mathrm{N}$, divided by the total number of subcarriers, which is also $\mathrm{N}$. Therefore the highest PAPR is:

$P A P R_{m}=\frac{N}{N}=\mathrm{N}$

This highest PAPR increases whenever the number of subcarriers increases. Thus, if $\mathrm{N} \rightarrow \infty=>\mathrm{X}_{\mathrm{k}}$ becomes Gaussian disseminated, for $\mathrm{k}=1, \ldots, \mathrm{N}$, which means that

$\mathrm{P}\left(X_{k}<P A P R_{m}\right)<1$

When the number of subcarriers tends to $\infty$ this probability gives

$\lim _{N \rightarrow \infty} \prod_{k=1}^{N} P\left(x_{k}<P A P R_{m}\right)=0$

If the above statement represents the probability of a signal $\mathrm{x}_{\mathrm{k}}$ to have a smaller PAPR than the given one $\mathrm{PAPR}_{\mathrm{m}}$, the likelihood of the signal to have a PAPR greater than $\mathrm{PAPR}_{\mathrm{m}}$ is

$\lim _{N \rightarrow \infty}\left(1-\prod_{k=1}^{N} P\left(x_{k}<P A P R_{m}\right)\right)=1$

Top of statement can be improved understood graphically in Figure 3, which shows the complementary cumulative distribution function (CCDF) of an OFDM signal. The CCDF denotes the probability of a signal to have a higher PAPR than a threshold PAPR, so in the figure, straight and straight up axes represent the threshold values of PAPR and the CCDF in that order. The CCDF simulation compares the CCDF of signals with dissimilar number of subcarriers (from 128 to 1024) and it corroborates the hypothesis. For all the unlike signals of the simulation, the probability of having higher PAPR than a fixed PAPR decreases as this fixed value 
increases. However, this probability increases as the number of subcarriers increases, and if $\mathrm{N}$ keeps mounting the probability tends to be 1 for several $\mathrm{PAPR}_{\mathrm{m}}$ so the PAPR of the signal when $\mathrm{N} \rightarrow \infty$ will always be greater than $P A P R_{m}$. The probability of having a peak higher than $\mathrm{PAPR}_{\mathrm{m}}$, though, decreases as $\mathrm{N}$ increases since the more subcarriers there are, the fewer probable it is that all they total carefully at the same moment, but still it may happen.

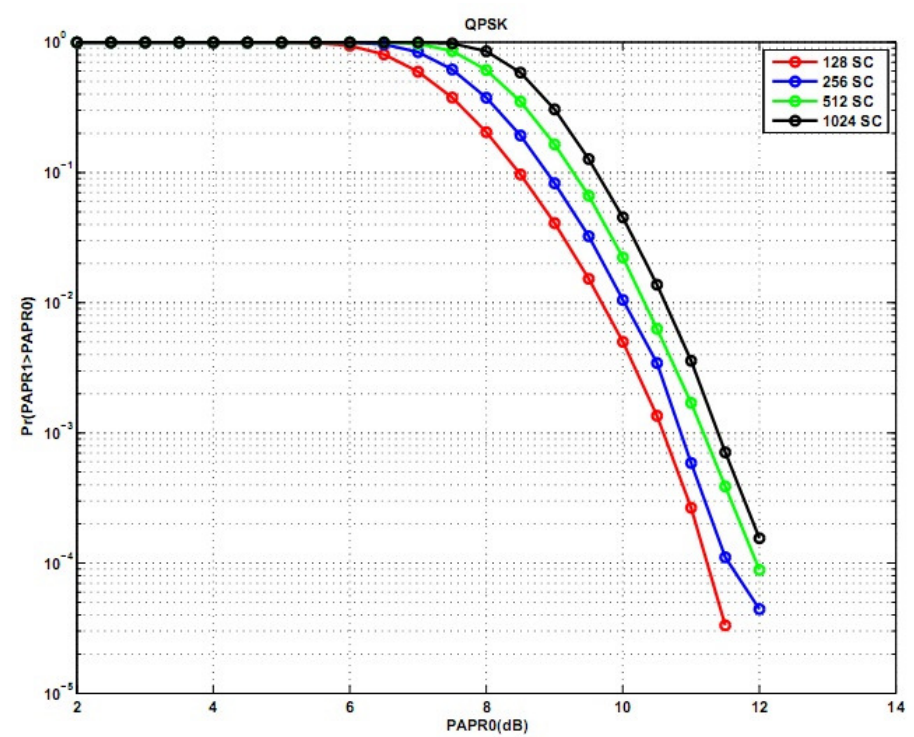

Fig 3: CCDF for different number of subcarriers

For different number of subcarriers the CDF is plotted in Figure 3, which has used QPSK modulation. It can be simply shown that higher number of carriers will be resulted into higher PAPR. OFDM has several properties, which make it a striking modulation scheme for high speed transmission links. However, one major difficulty is its large Peak to Average Power Ratio (PAPR). These large peaks cause saturation in power amplifiers, leading to inter modulation products among the subcarriers and troubling out of band energy. Therefore, it is desirable to reduce the PAPR. To reduce the PAPR, several techniques have been proposed such as clipping, coding, peak windowing, Tone Reservation and Tone Injection. But, most of these methods are unable to achieve concurrently a large reduction in PAPR with low complexity, with low coding overhead, without performance degradation and without transmitter receiver symbol handshake. The complex envelope of the OFDM signal, consisting of $\mathrm{N}$ carriers is given by,

$S_{\text {total }}=\sum_{k=-\infty}^{\infty} \sum a_{n, k} g(t-k T) e^{j n \frac{2 \pi}{T}} t$

Where $\mathrm{g}(\mathrm{t})$ is rectangular pulse of duration $\mathrm{T}$ and $\mathrm{T}$ is OFDM symbol duration. Peak to Average Power Ratio is defined by,

$P A P R=\frac{\max |S(t)|^{2}}{\varepsilon\left\{|S(t)|^{2}\right\}}$

From the central limit theorem, for large values of $\mathrm{N}$, the real and imaginary values of $\mathrm{S}(\mathrm{t})$ become Gaussian distributed. The amplitude of the OFDM signal there for has a Rayleigh distribution with zero mean and a variance of $\mathrm{N}$ times the variance of one complex sinusoid.

From this, it is seen that large PAPR occurs only infrequently.

Peak to Average power Ratio effect is described by Peak Envelop and Crest-Factor (CF) which is defined as the square root of Peak to Average Power Ratio. Here, we will refer it as Peak to Average power Ratio (PAPR).

\section{PAPR REDUCTION METHODS}

There have been many new approaches developed during the last few years. Several PAPR reduction techniques have been proposed in the literature. These techniques are divided into two groups.

\section{Active channels methods}

The data carrying channels will be affected here. So BER can be affected. But data throughput will be maximum.

Non Active channel methods

Here, the channels which are not carrying data will be used. So, data throughput will be decreased. But BER will not be affected. 


\section{Clipping Technique}

One of the simplest ways to reduce the PAPR is to clip the high amplitude peaks. This is a simplest technique used for PAPR reduction. Clipping means the amplitude clipping which restricts the peak envelope of the input signal to a preapproved value. Several clipping techniques have been described in the literatures. Some clip the signal at the output of the inverse discrete Furrier transform (IDFT). But consequent interpolation cause re-growth of the signal peaks. Other clipping techniques clip the signal after interpolation and use a filter to reduce the resulting out-of-band power. However the filters, which have been projected, are complicated and computationally classy. In addition they cause peak re-growth and result in significant distortion of the required signal. Clipping and filtering cause spectral splattering and initiate additional noise to the signal. The poverty of performance of this additional noise can be compensated by a smaller $\mathrm{A} / \mathrm{D}-/ \mathrm{D} / \mathrm{A}$ quantization noise due to a reduction of dynamical series by clipping.

Let $X[n]$ denote the pass band signal and $X_{c}[n]$ denote the clipped version of $X[n]$, which can be expressed as

$X_{c}[n]=\left\{\begin{array}{c}-A X[n] \leq-A \\ X[n]|X[n]|<A \\ A X[n] \geq A\end{array}\right.$

Where A is the pre-specified clipping level. However this technique has the following drawbacks:

- Clipping causes in-band signal distortion, resulting in Bit Error Rate performance degradation.

- It also causes out-of-band radiation, which imposes out-of-band interference signals to adjacent channels. This out-of-band radiation can be reduced by filtering.

- This filtering of the clipped signal leads to the peak re-growth. That means the signal after filtering operation may exceed the clipping level specified for the clipping operation.

\section{Tone Reservation Technique}

Common to all, OFDM systems has a large peak-to-average-power ratio (PAPR), which can lead to low power efficiency and nonlinear distortion at the transmit power amplifier. Tone reservation uses other vacant or kept tones to design a peak cancelling signal that lowers the PAPR of a transmit OFDM block. In contrast to previous methods, the new active-set method proposed here converges very quickly toward a minimum-PAPR solution at a lower computational cost. A well-organized real baseband algorithm is wellsuited for discrete multitone modulation (DMT) over twisted-pair copper wiring, where some sub-channels may have an inadequate SNR to reliably send data. The real PAPR problem occurs in the analog signal before the power amplifier, and results focus on this figure of merit. The performance of oversampling before applying PAPR reduction is analyzed, and results show that this is necessary to sufficiently handle the analog PAPR problem. An extension of the real-baseband technique can be applied to complex-baseband signals to help reduce PAPR in wireless and broadcast systems. By sacrificing 11 out of 256 OFDM tones (4.3\%) for tone reservation, over $3 \mathrm{~dB}$ of analog PAPR reduction can be obtained for a wireless system.

Fig 4: PAPR plot comparison of Original, Clipped and Clipped with TR

Figure 4 shows PAPR in $\mathrm{dB}$ for each symbol. It is obvious that clipping will reduce the PAPR for each symbol especially for the symbols having higher PAPR values. To reduce the PAPR further Non active channels will be

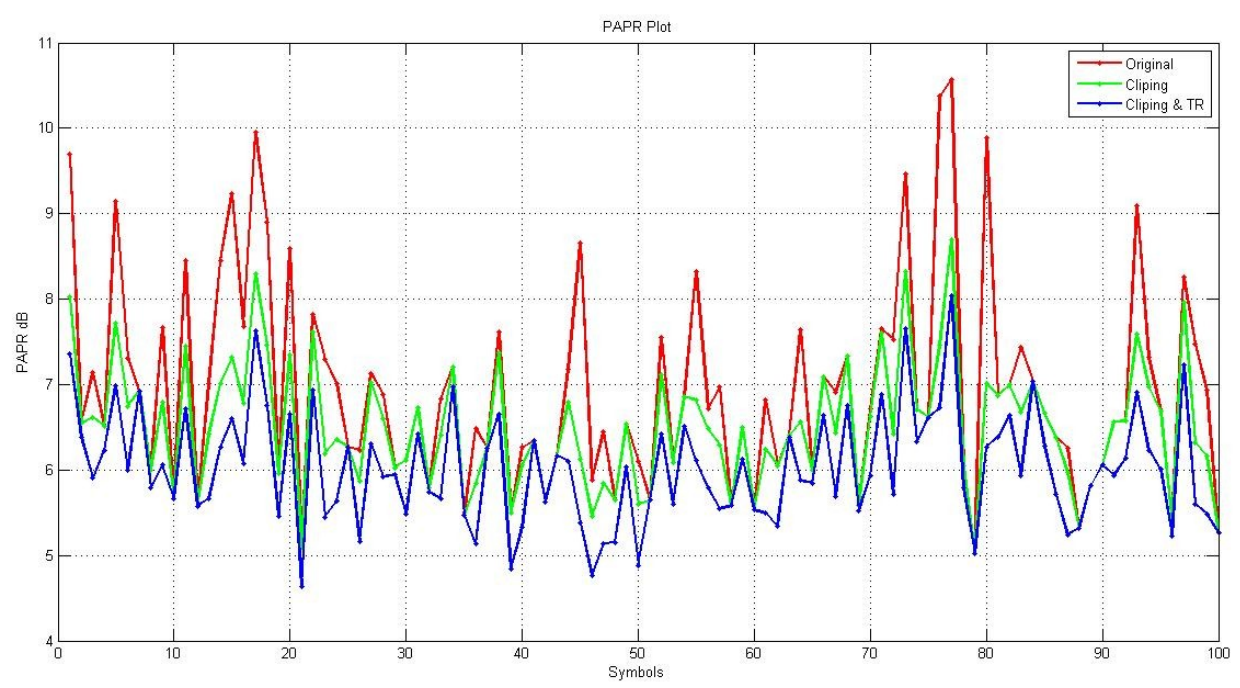


used. With help of Tone reservation method (Non Active channel method) PAPR will be reduced further. PAPR is within $4 \mathrm{~dB}$ to $10 \mathrm{~dB}$ for original signal. It is within $4 \mathrm{~dB}$ to $8.5 \mathrm{~dB}$ using clipping method. And it is within $4 \mathrm{~dB}$ to $8 \mathrm{~dB}$ for proposed method.

\section{CONCLUSION}

In the last years wireless communications have experienced a fast growth due to the high mobility that they allow. However, wireless channels have some disadvantages, like multipath fading, that make them difficult to deal with. A modulation that powerfully deals with selective fading channels is OFDM. But it has also disadvantages, like a high PAPR, that make it not so simple to implement.

Research is being done in order to reduce the effects of high PAPR in OFDM systems. Many techniques and algorithm are available to reduce the PAPR. These methods can be classified in Active channel methods and Non Active Channel methods. Tone reservation method for non-active channel is selected and Clipping for active channels is selected.

PAPR has been reduced up to $2 \mathrm{~dB}$ by applying both Clipping (active channel) and Tone reservation (non-active channel). Complexity will be increased here to combine both techniques. But significant reduction in PAPR has been achieved. Clipping will cause out of band distortion which can be reduced with filtering.

The high PAPR of OFDM systems causes the amplifier to work in the saturation region, making it to consume more power, but by proposed technique the high peaks are reduced. In this way the saturation region is avoided and power consumption is also reduced. More active channel methods can be combined with non-active channel methods for future expansion.

\section{Journal Papers:}

\section{REFERENCES}

[1] R. van Nee and R. Prasad, OFDM for Wireless Multimedia Communications, Artech House Publishers, Massachusetts, 2000.

[2] R. O'Neill and L. B. Lopes Envelop variation and spectral splatter in clipped multicarrier signals in Proc. IEEE PIMRc'95, vol. 1, pp. 71-75, Toronto, Canada, Sep. 1995.

[3] Mohammad Zavid Parvez Md. Abdullah Al Baki Peak To Average Power Ratio (PAPR) Reduction In OFDM Based Radio Systems Master Of Science Thesis Blekinge Institute Of Technology May 2010.

[4] A.H.M Shahparan Numerical Performance Evaluation Of PAPR Reduction In MIMO OFDM Wireless Communication Thesis Of Bachelor Of Science Department Computer Science \& Telecommunication Engineering, Noakhali Science Technological University,Sonapur.Nov-2011

[5] S. Hikmet, K. Georges, and J. Jsabelle, Transmission techniques for digital terrestrial TV broadcasting IEEE Comm. Magazine, pp.100-109, Feb. 1995

[6] T. A. Wilkinson and A. E. Jones Minimization of the peak to mean envelope power ratio in multicarrier transmission schemes by block coding in Proc. Of VTC'95, July 1995, pp. 825-831.

[7] D. J. G. Mestdagh, P. M. P. Spruyt, A Method to Reduce the Probability of Clipping in DMT Based Transceivers IEEE Trans. On Communications, Vol. 44, No. 10, Oct 1996, pp 1234-1238. 